Instituto Internacional de Investigación y Desarrollo Tecnológico Educativo INDTEC, C.A.

DOI: https://doi.org/10.29394/Scientific.issn.2542-2987.2020.5.17.18.328-349

OAI-PMH: http://www.indteca.com/ojs/index.php/Revista Scientific/oai

Ensayo Original / Original Essay

\title{
Torito de Pucará: Relación entre su producción y el desempeño académico estudiantil en Checca Pupuja
}

\author{
Autores: Pascual Martin Napa Bernuy \\ Universidad Nacional Mayor de San Marcos, UNMSM \\ pascualnapa20012001@gmail.com \\ Lima, Perú \\ https://orcid.org/0000-0002-5978-3375 \\ Gustavo Eduardo Vásquez Casahuaman \\ Universidad Nacional Mayor de San Marcos, UNMSM \\ gv720050@gmail.com \\ Lima, Perú \\ https://orcid.org/0000-0002-2746-1347 \\ Gabriel Andrés Roselló Sarrín \\ Universidad Nacional Mayor de San Marcos, UNMSM \\ gabriel.rosellosarrin@gmail.com \\ Lima, Perú \\ https://orcid.org/0000-0003-2159-9131
}

\section{Resumen}

El Torito de Pucará es una de las muestras de arte mestizo más representativas del Perú, esta cerámica no solo genera beneficios económicos para los artesanos sino también los provee de identidad y valores. Sin embargo, problemas principalmente en el desempeño académico de algunos niños, artesanos desde muy temprana edad, han llevado a suponer en algunos sectores de la población, que este problema se encuentra íntimamente relacionado con dichas actividades. En el presente ensayo nos dedicamos a estudiar este problema, partiendo por identificar el significado del Torito de Pucará, su elaboración, los actores que intervienen en ella y la relación que pueda tener con el desempeño académico de los niños dedicados a esta actividad. Nuestro objetivo, como quedará claro en el ensayo, es refutar tales aseveraciones y descubrir las verdaderas causas del problema como lo son la carencia de estrategias metodológicas educativas y, un mal monitoreo y acompañamiento en la práctica docente. Culminando por concebir a esta actividad artística no como una traba en el desarrollo académico de tales niños, sino como una oportunidad que habrá de ser aprovechada.

Palabras clave: arte; cerámica; desarrollo del niño; educación; patrimonio cultural.

Cómo citar este ensayo:

Napa, P., Vásquez, G., \& Roselló, G. (2020). Torito de Pucará: Relación entre su producción y el desempeño académico estudiantil en Checca Pupuja. Revista Scientific, 5(17), 328-349, e-ISSN: 2542-2987. Recuperado de: https://doi.org/10.29394/Scientific.issn.2542-2987.2020.5.17.18.328-349

Fecha de Recepción: 17-01-2020
Fecha de Aceptación: 23-04-2020
Fecha de Publicación: 05-08-2020 


\title{
Little bull of Pucara: Relationship between its production and student academic performance in Checca Pupuja
}

\begin{abstract}
El Little bull of Pucara is one of the most representative samples of mestizo art in Peru, this pottery not only generates economic benefits for artisans but also provides them with identity and values. However, problems mainly in the academic performance of some children, craftsmen from an early age, have led to suppose in some sectors of the population, that this problem is closely related to these activities. In the present essay we are dedicated to study this problem, starting by identifying the meaning of the Little bull of Pucara, its elaboration, the actors involved in it and the relationship it may have with the academic performance of children dedicated to this activity. Our objective, as will be clear in the essay, is to refute such assertions and discover the true causes of the problem, such as the lack of educational methodological strategies and poor monitoring and accompaniment in teaching practice. Culminating in conceiving this artistic activity not as an obstacle in the academic development of such children, but as an opportunity to be seized.
\end{abstract}

Keywords: art; ceramic art; child development; education; cultural heritage.

\footnotetext{
How to cite this essay:

Napa, P., Vásquez, G., \& Roselló, G. (2020). Little bull of Pucara: Relationship between its production and student academic performance in Checca Pupuja. Revista Scientific, 5(17), 328349, e-ISSN: 2542-2987. Recovered from: https://doi.org/10.29394/Scientific.issn.25422987.2020.5.17.18.328-349
}

Date Received:

17-01-2020
Date Acceptance: 23-04-2020
Date Publication: 05-08-2020 


\section{Introducción}

Mucha de la diversidad cultural que tiene el Perú es debido en gran parte a sus comunidades y culturas originarias, pero también debido a la fusión que hubo con otros grupos étnicos como los europeos y asiáticos. Una importante expresión de esta fusión es la cerámica de la comunidad Checca Pupuja, en el Departamento de Puno, en la cual participan desde los más pequeños hasta los más ancianos de dicha comunidad. Sin embargo, a diferencia de la antigüedad, ahora hay otras actividades que deben realizar los niños tales como estudiar y desarrollarse intelectual y profesionalmente. Por esta razón se exige normalmente que los niños no se dediquen a esta expresión artística ya que perjudica directamente sus estudios. De esta manera se pueden apoyar en la complicada situación educativa que enfrentan muchos alumnos de la zona. En este ensayo nos dedicaremos a resaltar y probar que tanto la educación y la cultura pueden trabajar en conjunto para una educación de calidad.

La motivación de la elaboración este ensayo surgió de las prácticas de lectura y escritura que el Profesor Rudy Mostacero implementó en la asignatura Lenguaje Académico II que cursamos en el segundo semestre del año 2019 en la Universidad Nacional Mayor de San Marcos, Lima, Perú, y como parte del primer año en la Escuela de Estudios Generales. El propósito era redactar un ensayo argumentativo que tuvo varios borradores, presentados y evaluados por los compañeros del curso y el profesor.

Es necesario hablar un poco de la historia del torito de Pucará, pues, contrariamente a lo que se piensa al ver el nombre, el torito de Pucará no se originó en Pucará, sino que proviene de la comunidad Checca Pupuja. Esta comunidad, como lo describe el Ministerio de Cultura (MINCUL, 2019a): se ubica en el distrito José Domingo de Choquehuanca, provincia de Azángaro, departamento de Puno y cuenta en la actualidad con aproximadamente 60 familias. La historia de esta comunidad se podría remontar desde los tiempos 
prehispánicos en la cual junto a otras comunidades conformaban la famosa cultura Pucará. Según el trabajo efectuado por el Ministerio de Comercio Exterior y Turismo (MINCETUR, 2013a): bajo el nombre de "Toro, torito de Pucará: Galería y estudios", presenta que con el tiempo esta comunidad se fue diferenciando y de acuerdo a los relatos se conocían por su seriedad y su palabra veraz y siempre cierta, por esta razón, se le empezó a llamar comunidad ch'equa, que tanto en quechua como en aymara que significa "verdad". Como el resto de las culturas que se asentaron en el altiplano, fueron conquistadas por los Incas y posteriormente sometidas por los españoles, su gente, aunque sometida, nunca dejó de luchar por su tierra. Posteriormente, en la República, serían víctimas de los abusos cometidos por los gamonales.

Nuestro propósito es demostrar la importancia y la relación que tiene esta producción artística con la educación. Con este fin, el desarrollo de nuestro ensayo argumentativo estará dividido en dos partes. La primera y más corta, se centrará en un aspecto descriptivo de la cerámica Checca Pupuja y pasaremos a revisar estudios anteriores con respecto al nivel de educación en la zona que rodea a la comunidad Checca Pupuja. Esta sección es sumamente importante para relacionar al lector con nuestro objeto de estudio, así como para que pueda entender tanto el punto de vista de la posición contraria como la nuestra. En la segunda parte, llevaremos a cabo la argumentación de nuestra tesis mediante estudios concernientes al tema de cultura y educación para exponer los argumentos y una contra argumentación. Finalmente, presentaremos las conclusiones.

\section{Desarrollo}

\subsection{Cerámica Checca Pupuja: un vistazo al torito de Pucará}

De acuerdo con la percepción de los pobladores del altiplano puneño se tiene entendido que el par de toritos de Pucará que se suele colocar en el techo de una casa simboliza la protección y felicidad en el hogar. Tal como lo 
explica Reyes (2015a): "es una dualidad andina que representa al marido y mujer que representan la fusión de energías positiva y negativa que busca el equilibrio y bien común" (pág. 14).

De igual manera, Reyes (2015b), describe que:

El acabado singular de los toritos son figuras imperfectas, de aspecto tosco, con aplicaciones de flores, elipses, capellones en el lomo, en el cuello como chalinas con aplicaciones geométricas zig zag y/o escalera, con orlas que le cuelgan del testuz. En fin la forma que adquiere en el uso común es como cuenco con un orificio a la altura del hueso sacro, todo ello con contenido de mayor valor estético y espiritual, conocidos también como qonopas (Quechua) y/o Illas (aymara) de uso ceremonial (pág. 9).

Todas estas decoraciones tienen un significado que según el Ministerio de Comercio Exterior y Turismo (MINCETUR, 2013b): su origen se remonta a la colonia, cuando en la fiesta de la Santísima Trinidad del mes de mayo se practicaba el señalakuy en el cual se escogía a un toro para sacrificarlo abriéndole un hueco por debajo del cuello y cortes en la frente. Esta ceremonia se daba como pago a la tierra y para la fecundidad, bajo la creencia de que lo semejante produce lo semejante. De acuerdo con Villegas (2008): detalla incluso que el torito de Pucará, la mayor expresión artística, tiene importancia como transposición cultural de lo español en lo andino. Por su parte, Stastny (1981): va un paso más allá afirmando que el toro fue incluido como parte de la mitología quechua, esto se evidencia con la consideración del toro como representación del Amaru. Como sostiene Reyes (2015c):

El torito moderno es un prototipo turístico, en sus acabados tienen más de dos colores chillones y brillantes llamativos para dar un atractivo artístico y comercial, generalmente se compra en pares. En un inicio fue símbolo de ceremonias, y ahora un tótem que le atribuyen poder de protección, cuando es colocada en los techos de las viviendas (pág. 15).

Como se puede deducir, la elaboración del torito de Pucará, requiere 
de un sofisticado y minucioso proceso, el cual implica un trabajo continuo y coordinado entre los miembros de un taller. El torito de Pucará, como lo señala el Ministerio de Comercio Exterior y Turismo (MINCETUR, 2013c); y el Ministerio de Cultura (MINCUL, 2019b): constituye una importante fuente de ingresos para muchas familias de Cuzco y Puno, principalmente porque este objeto viene siendo adquirido como un elemento decorativo y como protector del hogar.

Ante ello deducimos, que surge la necesidad de incrementar el número y calidad de los toritos; familias enteras suelen dedicarse a esta actividad productiva de sustento, en muchas de ellas también se ven implicados los niños del hogar, quienes adquieren responsabilidades en la producción y venta a una muy temprana edad. En el estudio del Ministerio de Comercio Exterior y Turismo (MINCETUR, 2013d), se describe que: "todos, varones, mujeres y niños son ceramistas por excelencia. Aprendiendo este arte desde su infancia mediante la educación informal, perfeccionándolo y especializándose a medida que avanzan los años" (pág. 42).

El comercio es también una actividad susceptible a este tipo de preparación, para el Ministerio de Comercio Exterior y Turismo (MINCETUR, 2013e):

Es cosa corriente ver a muchachos de diez e incluso echo [sic] años a cargo de todo un puesto de venta en los que se desempeñan con toda responsabilidad y seguridad recibiendo, de este modo, un entrenamiento que los capacita para el futuro (pág. 53).

\subsection{Situación educativa y estudios relacionados}

Es con respecto a este último punto donde surgen algunas polémicas e inquietudes puesto que no se concibe correcto, por parte de un sector de la población, que se involucre a los menores en estas actividades ya que los limita en sus actividades educativas y recreacionales. Vamos a revisar tres 
trabajos que trataron los problemas educativos desde tres perspectivas distintas:

\section{a). Benito Mamani Quispe: El problema con las matemáticas}

Los datos demuestran que el nivel educativo en los niños pertenecientes al distrito de Pucará, al igual que los de Pupuja (ambos dedicados principalmente a la cerámica) es realmente bajo. Acorde con esto, los resultados recogidos por Mamani (2018a): sobre la Evaluación Censal de Estudiantes del año 2015, llevada a cabo por el Ministerio de Educación a estudiantes de segundo grado, en el área de matemática, arrojó que solo un $3,8 \%$ se encuentra en el nivel satisfactorio, $10,3 \%$ en proceso, $43,6 \%$ en nivel de inicio y $42,3 \%$ en el nivel previo al inicio.

Así mismo, Mamani (2018b): reconoce tres principales causas de estos graves problemas educativos en la comunidad: una carencia de estrategias metodológicas de resolución de problemas matemáticos, un mal monitoreo y acompañamiento en la práctica docente y, una falta de acuerdos de convivencia.

En cuanto a la primera causa que expone Mamani (2018c), tiene mayor relevancia con el análisis realizado (por la relación con las actividades extracurriculares ejecutadas por los estudiantes), en este sentido: "[...] las estrategias metodológicas que utilizan los docentes son memorísticas y tradicionales [...]" (pág. 8); para poder mitigar este problema, se plantea utilizar "[...] estrategias que promuevan la capacidad de resolución de problemas matemáticos en los estudiantes, más no la resolución de únicamente ejercicios algebraicos y algorítmicos [...]" (pág. 9).

Es decir, Mamani (2018d), propone que:

[...] Se priorice el trabajo cooperativo entre estudiantes y en vez de desarrollar en las sesiones ejercicios algorítmicos y rutinarios, fundamentalmente enfatice la resolución de problemas del contexto real del estudiante como estrategia 
fundamental para el desarrollo de las competencias de esta área curricular (pág. 11).

De acuerdo con Iparraguirre (2009), citado en Mamani (2018e), complementa que: "se aprende matemática haciendo matemática, esto se produce en las interacciones del estudiante con el saber matemático, mediante la resolución de problemas" (pág. 16).

De todos estos aspectos podemos concluir que, como dice Mamani (2018f): "[...] las matemáticas se aprenden haciendo matemática y hacer matemática es saber resolver problemas diversos, por lo que, el desarrollo de esta área curricular debe partir de una situación problemática, lejos de desarrollar ejercicios rutinarios de carácter algorítmico" (pág. 16).

\section{b). Julio David Vilca: Las deficiencias en la comprensión lectora}

De igual manera, otro estudio realizado por Vilca (2018a): enfocado más en los resultados de comprensión lectora en los estudiantes de la institución educativa de Charquismo ubicada en la zona rural de Azángaro y dentro de la comunidad Checca Pupuja, lanza resultados preocupantes. En primer lugar, se encuentra la Evaluación Censal de Estudiantes (ECE) ejecutada en el año 2015. En el área de Comprensión lectora se obtuvo un $11 \%$, a diferencia del $25 \%$ obtenido el año anterior, lo cual posiciona al $65 \%$ del alumnado de esta institución en un estado de ineficiencia.

En segundo lugar, con respecto a las causas, Vilca (2018b), llega a conclusiones similares a las de Mamani, pues identifica tres causas como las responsables de tal desempeño como el: "[...] uso inadecuado de técnicas y estrategias de comprensión de lectura de los docentes, Desconocimiento del proceso de monitoreo, acompañamiento pedagógico y evaluación (MAE), y manejo de mecanismos negativos para promover acuerdos de convivencia escolar" (pág. 7). 
Una diferencia que tiene este caso con el anterior es que mientras que la aplicación de estrategias positivas en el área de matemáticas va a ayudar a una mejora del rendimiento académico de los estudiantes en esa asignatura; la aplicación de estrategias en la comprensión lectora, según Vilca (2018c): "[...] facilitarán un aprendizaje transversal para el resto de las otras áreas curriculares, $[\ldots]$ con técnicas y tácticas, uno tiene la gran posibilidad de poder comunicarse con facilidad con el resto del mundo, lo cual hace competente al lector" (pág. 12-13). Es decir, la solución de la problemática en este sector educativo tendrá un mayor campo de influencia que el anterior.

Evaluando esta situación, así como el campo de acción de la comprensión lectora que es más amplio, sus mecanismos de solución no solo se limitarían al ámbito educativo, sino que podrían remitirse al cultural, por ejemplo, materia de estudio en la siguiente parte. A modo de reflexión, Solé (2007), citado por Vilca (2018d): sugiere "involucrase dentro de un proceso de hacer inferencias y predicciones en forma interna y continua, encontrándose sostenida en la comunicación de los textos aportan experiencias propias" (pág. 4).

\section{c). Ceverino Machaca: La crítica a las estrategias aplicadas}

Por último, cabría hacer una breve mención al trabajo de Machaca (2018): el cual se enfocó no en una materia en específico, sino en la aplicación de los procesos pedagógicos y didácticos de los docentes en la institución educativa José Carlos Mariátegui. Entre sus conclusiones están:

- El director general de la institución requiere de un liderazgo pedagógico que le permita ejercer una buena gestión escolar (muy relacionado con la práctica constante del modelo de monitoreo, acompañamiento y evaluación (MAE).

- El hecho de no aplicar correctamente la gestión escolar y la convivencia 
en este ámbito son los principales factores para que se originen los problemas de aprendizaje de los alumnos.

- Se debe dejar de lado el modelo tradicional de enseñanza por uno que promueva más la interrelación del docente con los estudiantes, para que de esta manera las estrategias que éste aplique en su salón de clases tengan más probabilidad de éxito y establezca una confianza mutua y una comunicación asertiva.

Hay una importante razón por la cual hemos empezado entablando los dos temas concernientes a nuestro ensayo de manera separada. Tanto la descripción de la cerámica Checca Pupuja como el análisis de la situación educativa actual de los estudiantes en la comunidad nos permiten tener un panorama completo de la problemática que se forma en torno a ambos aspectos.

Incluso, de manera aislada, se pueden inferir ciertas relaciones que podrían existir entre la educación y la cultura en este singular caso. Ahora la pregunta sería: ¿Los niños deberían dejar de hacer el adiestramiento en cerámica para concentrarse solamente en estudiar?; ¿Es el tiempo el principal factor por el cual el rendimiento académico es tan bajo? No son preguntas fáciles de responder, y más ahora que a inicios de este año se ha declarado por el Ministerio de Cultura (MINCUL, 2019c): como Patrimonio Cultural de la Nación a los "conocimientos, técnicas y prácticas asociadas a la producción de cerámica tradicional de la comunidad de Checca Pupuja" (pág. 7).

\subsection{Argumentación de la tesis}

\subsubsection{Relación entre educación y cultura}

Es necesario hacer un análisis de la relación entre cultura y educación para poder entender este problema, sus características e importancia. De lo 
que dicen Colom y Carles (1997a), citados por Herrera (2007a): se deduce que la actual sociedad contemporánea posmoderna ha puesto en crisis a ésta misma por una falta de metas al progreso y valores causada por la continua intromisión de la economía de mercado en la vida cotidiana. Esta degradación en la cultura apoyada por la globalización y la comunicación en redes sociales acelera el paso a un mundo menos humano, lo cual afecta también el rol de la educación el cual debería consistir en prevenir estos procesos en la sociedad y en la cultura.

Por otro lado, Giroux (1994a), citado por Herrera (2007b): sostiene que el problema principal de esta sociedad es la economía de consumo que se intensifica con el pasar del tiempo. A su vez, sostiene que la educación todavía no está del todo supeditada a este consumismo a diferencia de lo que afirman Colom y Carles en el anterior parágrafo. Por eso, Giroux (1994b), citado en Herrera (2007c): agrega que, además de la capacidad que ha desarrollado el sistema empresarial para agilizar y masificar sus ventas y así aumentar significativamente sus ganancias, también se añade un nuevo panorama social.

Este proceso que solo perpetúa la degradación de la sociedad, su cultura y los valores no es para nada favorable para la educación que, es cierto, se ve de muchas maneras influenciada por la sociedad posmoderna en detrimento de los valores y la cultura. Por eso podemos concluir en esta sección que un importante paso para revertir este proceso es poner en sintonía a la cultura y a la educación y no solo con cambios en las mallas curriculares.

\subsubsection{Argumentos}

Según los estudios mostrados se ve una clara deficiencia en la educación de los niños de la zona rural de Azángaro y dentro de la comunidad Checca Pupuja. Así que vamos a presentar argumentos a favor de que la elaboración por parte de los niños de la cerámica del torito de Pucará, no es 
la verdadera razón por la cual su educación se ve afectada:

\section{a). Primer argumento}

Partiendo de la historia del Perú, siempre se ha tendido a mostrar aires centralistas que junto a las grandes migraciones del campo a la ciudad ha dejado más que evidente el estado de abandono del Estado hacia las zonas alejadas de la capital, como es el caso de Puno, que es el departamento donde se encuentra la comunidad Checca Pupuja. Teniendo como grandes deficiencias los sectores más importantes de la población, los cuales son salud y educación, se cree que este último ha sido víctima del ya mencionado olvido por parte del Estado, siendo esta la principal razón por la cual se dan los alarmantes resultados de los censos realizados en esta comunidad. Dejando de lado la posibilidad de que estos bajos resultados sean a causa de las actividades de los niños en torno a la cerámica del torito de Pucará, ya que hay muchos más departamentos en donde no se realizan estas actividades, pero igual se ven los mismos o peores resultados.

Es así, que tenemos nuestro primer argumento que viene a ser que, con un sistema de enseñanza eficiente, los niños elaboradores de la cerámica del Torito de Pucará, no se vean afectados en la educación. De esta forma, se apoya en las tres principales causas que identificaba Mamani anteriormente.

No se puede echar la culpa a la expresión cultural, como si fuese el factor negativo que impacta la situación educativa, ya que el factor más evidente según estos estudios son las estrategias utilizadas por los docentes y la gestión escolar.

\section{b). Segundo argumento}

En el segundo argumento, tenemos que los niños que elaboran la cerámica del Torito de Pucará, no pueden dejar de realizar esta actividad ya que les ha otorgado beneficios directos en los distintos campos de la 
educación ¿En qué campos y en qué sentidos? Pues por ejemplo, en los cursos académicos de Historia, Lenguaje y Matemática, ya que al ser la elaboración de la cerámica del torito una actividad económica, estos niños se ven envueltos en la historia (la historia del torito de Pucará y su significado), la comunicación con el mundo por parte de los turistas que llegan a la comunidad Checca Pupuja, en busca de los toritos y con la matemática, ya que son piezas valiosas de cerámica artesanal destinadas a los turistas peruanos e internacionales.

El entorno social que genera la elaboración del torito permite al infante generar nuevo conocimiento en matemática, comprensión lectora, etc., relacionándolo con el conocimiento previo adquirido en su comunidad. Señalando a Quintero (2019): "[...] el individuo modifica las estructuras conceptuales que ha adquirido previamente a través de la interacción social y aplica los procesos cognitivos necesarios para cimentar las bases de un nuevo aprendizaje [...]" (pág. 331).

\subsubsection{Contraargumentación}

El tema de la relación entre la cultura y educación no es sencillo y, así como ha habido autores que exponen argumentos a favor, también hay quienes han estado en contra. Uno de estos investigadores, es Levison (1991), citado por Salazar (1994a), quien afirma que: "la participación de los niños en actividades económicas y educativas se rige por la situación económica del hogar, aunque también inciden la demanda de trabajo infantil y la oferta de educación pública” (pág. 5).

Una buena idea de la relación entre la educación y la explotación infantil, nos la da el siguiente extracto de Krohn-Hansen (1990), citado por Salazar (1994b):

Como se acaba de mencionar, la relación entre la escuela y el trabajo infantil varía según se de en regiones urbanas o rurales, 
Instituto Internacional de Investigación y Desarrollo Tecnológico Educativo INDTEC, C.A. DOI: https://doi.org/10.29394/Scientific.issn.2542-2987.2020.5.17.18.328-349

OAI-PMH: http://www.indteca.com/ojs/index.php/Revista_Scientific/oai

Ensayo Original / Original Essay

y de acuerdo a contextos socioeconómicos y geográficos, lo mismo que según tipos de familias. [...] Así las niñas hacen menos tareas propiamente agrícolas y los niños menos tareas domésticas, lo que no significa que las niñas no colaboren ampliamente en las primeras. En comparaciones con el trabajo de los adultos, se ha encontrado que los niños alcanzan a trabajar casi el mismo tiempo que los adultos a edades de nuevo muy tempranas y con el mismo grado de eficiencia en tareas exigentes como el deshierbe y el arado de la tierra (pág. 7).

Las necesidades del infante para trabajar pueden provenir de dos factores: el primero es la necesidad colectiva familiar la cual a su vez se puede deber a rasgos económicos y culturales. El segundo factor consiste, como bien dice Salazar (1994c): en las "necesidades individuales que se traducen en configuraciones empíricas observables en distintos arreglos interfamiliares" (pág. 5).

Podemos reconocer como las causas más importantes del trabajo en menores la pobreza y los patrones culturales propios de la familia. Estas causas también se encuentran en el caso de los menores de la comunidad Checca Pupuja, pues estos tienen como una de sus principales fuentes de ingreso y subsistencia la elaboración de estas cerámicas. Sin embargo, es necesario mencionar que en esta comunidad existe una fuerte relación cultural-axiológica en cuanto a la elaboración de los toritos de Pucará. Esta actividad productiva forma parte de su propia cultura, valores, creencias y costumbres.

El torito de Pucará, como hemos visto en la primera parte de este ensayo, es significativo pues cumple distintas funciones, sean la protección a los hogares de estas familias, valores religiosos totémicos, símbolo de identificación cultural y también como mecanismo de subsistencia. Por ende, privar a estos niños de una actividad propia de su cultura, ocasionaría, más bien, privarlos de su identidad y valores. 
Instituto Internacional de Investigación y Desarrollo Tecnológico Educativo INDTEC, C.A.

DOI: https://doi.org/10.29394/Scientific.issn.2542-2987.2020.5.17.18.328-349

OAI-PMH: http://www.indteca.com/ojs/index.php/Revista_Scientific/oai

Ensayo Original / Original Essay

Estudios recientes muestran la posibilidad de articular habilidades artísticas con el aprendizaje de materias académicas. En ese sentido, es posible empezar a ver la elaboración de toritos, por parte de menores de la comunidad Checca Pupuja, como una oportunidad más que como un problema o traba en su desarrollo escolar y familiar. Estas premisas están presentes en el trabajo expuesto por Zambrano (2016a), en el que se expresa literalmente lo siguiente:

La cerámica les permite a los niños el desarrollo mental y emocional. La cerámica es una actividad dinámica, esta cumple un papel muy importante en los niños. Los niños plasman todas sus sensaciones y a la vez nos permite apreciar las creaciones que el niño nos deja plasmado en su labor. A nivel psicológico es muy terapéutica, es de gran ayuda para los niños agresivos, porque la buscan espontáneamente y la usan con vigor, y les ayuda a descargar malas energías, en los niños tímidos el trabajar en grupo con arcilla le permite compartir con sus compañeros y conocerlos, despierta en ellos sentimientos de compañerismo y así aprenden a trabajar en grupo. Ha sido comprobado que los niños disfrutan mucho realizar el modelado en arcilla, dejar plasmada su huella, la construcción de un objeto en ellos es muy gratificadora (pág. 17).

En esa misma línea, Zambrano (2016b), menciona que: "el uso de la motricidad fina en los niños, les permite desarrollar sus estímulos. La motricidad fina crea en el estudiante, hasta el más tímido, un desenvolvimiento más creativo, habilidad en la comunicación y participación en el aula" (pág. $34)$.

Interpretando a Iwai (2002a): se concluye que las actividades artísticas o "educación artística", como este autor las llama, permiten a los niños una mejor interacción y conducta con su entorno social, dándoles, además, herramientas de comunicación adecuadas. Para reforzar su punto de vista se apoya en argumentos formulados por la Association for the Advancement of Arts Education (AAAE, 2001), citado por Iwai (2002b): y pone de manifiesto 
que: "es posible mejorar las aptitudes interpersonales de los niños preparándolos para el trabajo en equipo, inculcándoles la tolerancia y la valoración de la diversidad de los individuos y las ideas, y habilitándolos para comunicarse con eficacia" (pág. 6).

\section{Conclusiones}

De los diversos resultados que hemos visto durante nuestra exposición y análisis de argumentos podemos llegar a las siguientes conclusiones finales. Cualquier estudio pedagógico en la actualidad no debe limitarse solamente al ámbito curricular, sino que debe tomar en cuenta otras dimensiones extracurriculares tales como en nuestro caso hemos expuesto, la cultura. Hay una relación profunda y compleja entre la educación y la cultura que, si se pasa por alto, se comete el error de percibir a la cultura como un obstáculo académico de los estudiantes.

Parte de la culpa de la situación de los estudiantes de la zona circundante a la comunidad Checca Pupuja se debe en gran medida a aspectos de carácter pedagógico (en el sentido del inadecuado empleo de estrategias) y administrativo (referente a la gestión que se hace de la institución y el monitoreo que el Estado realiza sobre esta). Por lo tanto, no es válido el sostener que los niños deberían abandonar aquella tarea que los provee de una identidad y cultura por problemas ajenos a esta actividad.

Más que una amenaza, hay que ver en estos niños ceramistas una doble ventaja, tanto como proveedores de identidad y cultura como de contribuir a lograr una mejor calidad de vida para sus familias. Parte de una propuesta propia sería que los docentes intenten relacionar las habilidades que los niños desarrollan como ceramistas con los aprendizajes escolares, de esta manera se obtendrá un mejor rendimiento en sus estudios. Transformar esta práctica en una estrategia formal en las instituciones podría traer resultados favorables tanto para los niños y las familias como para la 
comunidad.

Tomando en cuenta el anterior señalamiento se puede refutar la afirmación de que la única razón por la que los niños y jóvenes realizan la elaboración de la cerámica es por tradición o por mantener a su familia. Esta tarea no es una traba para el desarrollo personal y profesional del joven, sino que logra incluso mejorar los aspectos emocionales, interpersonales, sociales, artísticos e identitarios de la comunidad Checca Pupuja.

\section{Referencias}

Herrera, L. (2007a,b,c). La educación y la cultura: Una lectura y propuesta desde la filosofía de la praxis. Sophia: Colección de Filosofía de la Educación, (1), 187-231, ISSN: 1390-3861; e-ISSN: 1390-8626. Recuperado de:

https://dialnet.unirioja.es/servlet/articulo?codigo $=5973014$

Iwai, K. (2002a,b). La contribución de la educación artística a la vida de los niños. Perspectivas: Revista trimestral de educación comparada, XXXII(4), 1-18, e-ISSN: 0304-3053. Recuperado de:

http://www.ibe.unesco.org/sites/default/files/resources/prospects124 spa.pdf\#page $=25$

Machaca, C. (2018). Aplicación de procesos pedagógicos y didácticos por los docentes de la IEP № 72006 José Carlos Mariátegui - José Domingo Choquehuanca - Azángaro - Puno. Tesis. Lima, Perú: Escuela de Postgrado de la Universidad San Ignacio de Loyola. Recuperado de:

http://200.37.102.150/bitstream/USIL/8492/4/2018 MACHACA RAMO S CEVERINO HERMOGENES.pdf

Mamani, B. (2018a,b,c,d,e,f). Trabajo colegiado para mejorar los aprendizajes de matemática de los estudiantes de la IES José Domingo Choquehuanca - Azángaro. Tesis. Lima, Perú: Escuela de 
Instituto Internacional de Investigación y Desarrollo Tecnológico Educativo INDTEC, C.A.

DOI: https://doi.org/10.29394/Scientific.issn.2542-2987.2020.5.17.18.328-349

OAI-PMH: http://www.indteca.com/ojs/index.php/Revista_Scientific/oai

Ensayo Original / Original Essay

Postgrado de la Universidad San Ignacio de Loyola Recuperado de: http://200.37.102.150/bitstream/USIL/8107/4/2018 MAMANI QUISPE BENITO.pdf

MINCETUR (2013a,b,c,d,e). Toro, torito de Pucará: Galería y estudios. ISBN: 978-612-45764-0-09. Lima, Perú: Ministerio de Comercio Exterior y Turismo; Reimpresión por Editora Diskcopy SAC.

MINCUL (2019a,b,c). Declarar Patrimonio Cultural de la Nación a los Conocimientos, técnicas y prácticas asociadas a la producción de cerámica tradicional de la comunidad de Checca Pupuja. Resolución Viceministerial № 027-2019-VMPCIC-MC. Lima, Perú: Ministerio de Cultura. Recuperado de:

https://www.gob.pe/institucion/cultura/normas-legales/265556-0272019-vmpcic-mc

Quintero, K. (2019). Transformación del Aprendizaje desde el Enfoque Social (TADES). Revista Scientific, 4(13), 322-334, e-ISSN: 25422987. Recuperado de: https://doi.org/10.29394/Scientific.issn.25422987.2019.4.13.17.322-334

Reyes, F. (2015a,b,c). La simbología totémica del torito de Pucará. Perú: Sitio Web del Colegio de Antropólogos del Perú. Recuperado de: https://www.arqueologiadelperu.com/la-simbologia-totemica-del-toritode-pucara/?print=pdf

Salazar, M. (1994a,b,c). La significación social del trabajo infantil y juvenil en América Latina y el Caribe. Revista Colombiana de Educación, (28), 1-19, ISSN: 0120-3916; e-ISSN: 2323-0134. Recuperado de: https://doi.org/10.17227/01203916.5360

Stastny, F. (1981). Las artes populares del Perú. ISBN: 84-499-4690-5. Madrid, España: Fundación del Banco Continental para el Fomento de la Educación y la Cultura (EDUBANCO); Producción a cargo de Alianza Editorial, S.A. Recuperado de: https://fundacionbbva.pe/wp- 
Instituto Internacional de Investigación y Desarrollo Tecnológico Educativo INDTEC, C.A.

DOI: https://doi.org/10.29394/Scientific.issn.2542-2987.2020.5.17.18.328-349

OAI-PMH: http://www.indteca.com/ojs/index.php/Revista_Scientific/oai

Ensayo Original / Original Essay

content/uploads/2016/04/libro 000052.pdf

Vilca, J. (2018a,b,c,d). Capacitación docente para mejorar los niveles de aprendizaje en comprensión de lectura en estudiantes de la IEP. 72693 - Charquismo - Azángaro. Tesis. Lima, Perú: Escuela de Postgrado de la Universidad San Ignacio de Loyola. Recuperado de: http://200.37.102.150/bitstream/USIL/8332/4/2018 VILCA JUSTO JU LIO DAVID.pdf

Villegas, F. (2008). José Sabogal y el Arte Mestizo: El Instituto de Arte Peruano y sus Acuarelas. Tesis. Lima, Perú: Facultad de Letras y Ciencias Humanas E.A.P. de Arte de la Universidad Nacional Mayor de San Marcos. Recuperado de:

http://200.62.146.130/bitstream/handle/cybertesis/4178/Villegas tl.pdf ?sequence $=1$ \&isAllowed $=\mathrm{y}$

Zambrano, S. (2016a,b). La cerámica y la motricidad fina en los estudiantes de educación inicial paralelo "A" del jardín "Dolores Veintimilla de Galindo" de la ciudad de Riobamba, provincia de Chimborazo, parroquia Velasco, cantón Riobamba, durante el año lectivo 2015-2016". Tesis. Riobamba, Ecuador: Universidad Nacional de Chimborazo. Recuperado de:

http://dspace.unach.edu.ec/bitstream/51000/1589/1/UNACH-FCEHTTG-C.ESTE-000001.pdf 
Ensayo Original / Original Essay

\section{Pascual Martin Napa Bernuy \\ e-mail: pascualnapa20012001@gmail.com}

Nacido en Lima, Perú, el 27 de octubre del año 2001.

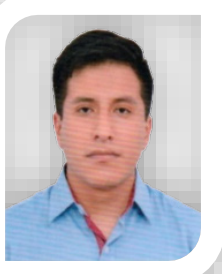
Estudiante de segundo año de la Carrera de Derecho en la Facultad de Derecho y Ciencias Políticas de la Universidad Nacional Mayor de San Marcos (UNMSM). 
Ensayo Original / Original Essay

\section{Gustavo Eduardo Vásquez Casahuaman}

e-mail: gv720050@gmail.com

Nacido en Lima, Perú, el 25 de julio del año 2001.

Estudiante de segundo año de Comunicación Social en la Facultad de Letras y Ciencias Humanas de la Universidad Nacional Mayor de San Marcos (UNMSM). 


\section{Gabriel Andrés Roselló Sarrín} e-mail: gabriel.rosellosarrin@gmail.com

Nacido en Lima, Perú, el 25 de abril del año 1998.

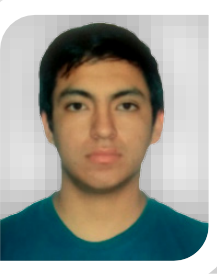
Estudiante de segundo año de la Escuela Profesional de Educación Física de la Universidad Nacional Mayor de San Marcos (UNMSM). 